Recebido em 04/2016. Aceito para publicação em 01/2019.

\title{
SISTEMA ESPECIALISTA APLICADO AO DIAGNÓSTICO DE FALHAS EM REFRIGERADORES DOMÉSTICOS
}

\section{SPECIALIST SYSTEM APPLIED TO FAULT DIAGNOSIS IN HOUSEHOLD REFRIGERATORS}

\author{
Antonio Carlos Barbosa Zancanella ${ }^{1}$ \\ Rômulo Maziero² \\ Juan Carlos Campos Rubio ${ }^{3}$
}

Resumo: O presente trabalho teve como objetivo o desenvolvimento de um sistema especialista para uso como tutor inteligente, auxiliando no treinamento de técnicos em refrigeração. Foram utilizadas técnicas, como Diagrama de Ishikawa, Análise da Árvore de Falhas (FTA) e Análise dos Modos de Falha e seus Efeitos (FMEA), para o levantamento dos principais modos de falhas e procedimentos de manutenção corretiva. O conhecimento heurístico foi programado em um sistema especialista, utilizando a plataforma CLIPS. O programa interage com o usuário, fornecendo o caminho para correção da falha no refrigerador. Observou-se que o sistema especialista é uma excelente ferramenta para armazenamento de informações heurísticas, podendo ser utilizado como ferramenta de apoio às tarefas de manutenção corretiva básica, como tutor inteligente ou evoluir em incrementos, possibilitando um aperfeiçoamento contínuo do programa. Palavras-chave: FTA; FMEA; Tutor inteligente; CLIPS.

Abstract: The present work had as objective the development of a specialist system for use as intelligent tutor, assisting in the training of technicians in refrigeration. We used techniques such as Ishikawa Diagram, Fault Tree Analysis (FTA) and Failure Modes Analysis and their Effects (FMEA) to investigate the main failure modes and corrective maintenance procedures. The heuristic knowledge was programmed into a specialist system using the CLIPS platform. The program interacts with the user providing the path for fault correction in the refrigerator. It was observed that the expert system is an excellent tool for storing heuristic information, and it can be used as a tool to support basic corrective maintenance tasks such as intelligent tutor, or evolve in increments, allowing a continuous improvement of the program.

Keywords: FTA; FMEA; Intelligent tutor; CLIPS.

\section{INTRODUÇÃO}

Os refrigeradores domésticos são uma necessidade da sociedade moderna, fazem parte do cotidiano das pessoas. Com o crescente apelo energético e ambiental, uma manutenção adequada pode reduzir o consumo de energia e assegurar maior confiabilidade ao sistema de refrigeração.

O conhecimento técnico sobre manutenção de refrigeradores fica a cargo de empresas, que, em muitos casos, não possuem procedimentos sistemáticos de

\footnotetext{
1 Instituto Federal de Educação, Ciência e Tecnologia do Espírito Santo - IFES, Brasil. E-mail: antonio.zancanella@ifes.edu.br.

2 Universidade Federal de Minas Gerais - UFMG, Brasil. E-mail: maziero@ufmg.br.

3 Universidade Federal de Minas Gerais - UFMG, Brasil. E-mail: juan@ufmg.br.

Revista Univap - revista.univap.br

São José dos Campos-SP-Brasil, v. 25, n. 47, jul. 2019. ISSN 2237-1753
} 
manutenção, como, por exemplo, um sistema especialista (SE). Um SE é um conhecimento específico sobre um domínio de aplicação particular e limitado sistematizado em um software, este deve ser atualizado e melhorado, tornando-se cada vez mais preciso. O conhecimento representado em um SE é de especialista de um domínio de conhecimento, parte desse conhecimento é fundamentado em relações causa efeito. Essas relações ou regras práticas foram adquiridas pelo especialista ao longo da carreira profissional e podem possuir natureza heurística.

As experiências heurísticas representam um conhecimento informal, que permitem a um especialista rapidamente pesquisar a solução de um problema sem ter que realizar uma análise detalhada de uma situação particular, porque ou uma análise de um problema similar já foi feito ou uma relação já foi aprendida de experiências passadas (SILVA, 2015).

Como vantagem em utilizar sistemas especialistas tem-se o aumento da disponibilidade de conhecimento: conhecimento pode ser socializado, ajudando mais pessoas a se aperfeiçoarem em determinado domínio específico; permanência do conhecimento: o conhecimento armazenado em um sistema especialista pode ser utilizado por várias pessoas e pode ser mantido em uma instituição como conhecimento agregado; resposta rápida e atuação como um tutor inteligente para treinamento e/ou consulta e banco de dados inteligente que fornece explicação sobre solução de problemas em detalhes.

O conteúdo sistematizado, no sistema especialista, pode ser usado como ferramenta de apoio didático, para treinamento e apoio direto no processo de manutenção, proporcionando uma ferramenta com resultados constantes, independente das pressões exercidas pelo ambiente de trabalho, assim a solução final apoiada pelo sistema especialista é segura.

Nesse contexto, o objetivo do trabalho foi sistematizar o conhecimento sobre manutenção de refrigeradores domésticos em um sistema especialista e ser utilizado como um tutor inteligente para discentes técnicos em mecânica.

\section{DESENVOLVIMENTO}

\subsection{Sistema fundamentado em conhecimento}

Para construção do sistema especialista foi escolhido o software CLIPS, a primeira versão do software foi criada pela Johnson Space Center da NASA, em 1985, o software é uma ferramenta conhecida como SHELL, isso significa que a máquina de inferência do sistema já é parte do software, o usuário entra apenas com o conhecimento na modelagem, a máquina de inferência tem função de modelar o processo cognitivo humano. Segundo Giarratano e Riley (1994), CLIPS é uma linguagem de programação multiparadigma a qual fornece suporte à programação tradicional, ou seja, baseia-se em 
procedimentos, programação orientada a objetos e regras.

Segundo Silva (2015), as principais etapas da criação de um sistema especialista são: análise da viabilidade; aquisição do conhecimento; representação do conhecimento; implementação; verificação e validação e documentação.

$\mathrm{Na}$ etapa de viabilidade, identificou-se utilidade do sistema especialista e se é possível criar um sistema para o tipo de problema proposto. Como o conhecimento modelado pode ser representado sem grandes necessidades de recursos visuais e auditivos a implementação do sistema especialista foi possível de ser realizada. A segunda etapa consistiu em aquisição do conhecimento, na qual foram utilizadas as seguintes fontes de conhecimento: artigos; livros e especialistas da área.

Para aquisição de conhecimento inicial sobre os modos de falha, foi utilizada a técnica de Ishikawa. Para aquisição e sistematização do conhecimento, foi utilizada a técnica de Análise dos Modos de Falha e seus Efeitos (FMEA). Esta técnica possui a característica de ser uma técnica dinâmica para diagnósticos de falhas. Na etapa de representação do conhecimento, escolheu-se a técnica de Análise da Árvore de Falhas (FTA), nessa as causas raízes funcionam como regras que desencadeiam eventos superiores na árvore de falha. Foi definido que o sistema trabalharia com o parâmetro de entrada sendo o diagnóstico da falha, por meio de uma medida a ser realizada no sistema de refrigeração pelo usuário (por exemplo, corrente elétrica no compressor) dado o diagnóstico como saída; ou seja, a saída foi estabelecida como sendo o procedimento de manutenção adequado para uma dada causa. Foi gerado um pseudocódigo, no qual estrutura o funcionamento do sistema em perguntas e possíveis respostas. O usuário final do sistema é um técnico em mecânica com conhecimentos básicos em refrigeração, o pré-requisito, para uso do SE, é possuir conhecimento em mecânica (preferência em nível técnico) e possuir algum conhecimento prévio em refrigeração.

O SE funciona basicamente como um diagnóstico médico, no qual o médico detecta a causa da doença (por meio de alguma medição) e fornece o tratamento adequado, este mesmo tipo de raciocínio foi aplicado ao sistema técnico, em que as causas tomadas como prioritárias foram as indicadas pelo especialista da área como as mais frequentes.

Foi desenvolvido código em clipes, o código foi com base em regras, as regras são ativadas por fatos, por exemplo, a regra substituir o compressor é disparada quando detecta-se falha pelo usuário compressor. O desenvolvimento seguiu uma metodologia incremental, ou seja, foram implementadas algumas funções iniciais, em futuras revisões novas falhas podem ser implementadas ao sistema, esta característica de aprimoramento dinâmico de um SE fez com que as técnicas de FMEA e FTA fossem adequadas para auxiliar a resolução do problema. É importante observar que um sistema especialista pode nunca ficar pronto; no entanto, com novos incrementos de 
funções, ele é capaz de auxiliar na resolução de uma gama maior de problemas. O incremento de funções no sistema foi previsto para ser feito de forma manual, ou seja, a cada atualização o engenheiro responsável pelo SE adiciona novos modos de detecção de falha, por meio da atualização do código. $O$ código inicial foi pensado de maneira que fosse fácil inserir novas funções.

Por fim, foi realizada a verificação e validação do sistema. O sistema possui, inicialmente, 17 eventos de entrada, todos independentes. Esses eventos são falhas no sistema de refrigeração, cada consulta ao SE pode fornecer a solução de apenas uma falha, sendo assim, o sistema pode apresentar 17 combinações de cenários de saída, felizmente para verificar todas as saídas possíveis basta verificar o programa, pois cada uma dessas saídas é mostrada separadamente. Como existem apenas 17 tipos de saídas, basta verificar cada uma. Esta abordagem teve como intuito modelar o raciocínio de um especialista humano que trabalha corrigindo as falhas uma a uma, ordenando a tarefa de detecção das mais triviais para as mais complexas. Para validação do sistema especialista, ele foi testado por especialistas da área, que forneceram críticas construtivas para futuras implementações.

\subsection{Diagrama de Ishikawa aplicado ao sistema de refrigeração}

Com objetivo de realizar um levantamento inicial das causas de falhas, foi utilizada a técnica de Ishikawa, o sistema refrigerador foi dividido em três partes básicas, sistema térmico, sistema de controle e isolamento. A Figura 1 mostra as principais causas de falhas associadas ao sistema térmico do refrigerador.

Cada falha do sistema térmico está associada a um item do refrigerador, o item mais complexo é o compressor. Para um melhor entendimento da falha no compressor, foram levantadas as causas de falha utilizando a técnica de Ishikawa. A Figura 2 mostra as principais causas de falha no compressor, estas podem ser divididas em mecânicas e elétricas, as primeiras cinco causas, de cima para baixo, representam componentes relacionados ao sistema mecânico, a última causa é de natureza elétrica, por vez essas causas poderiam ser estudadas com mais detalhes, no entanto, considerou-se este estudo como fora do escopo inicial. As falhas relacionadas ao motor elétrico podem ter origens em outras partes do sistema de refrigeração, por exemplo, no filtro secador, se existir umidade em excesso passando pelo compressor, o motor elétrico será danificado devido à corrosão dos componentes. 
Figura 1 - Possíveis causas de falha no sistema térmico.

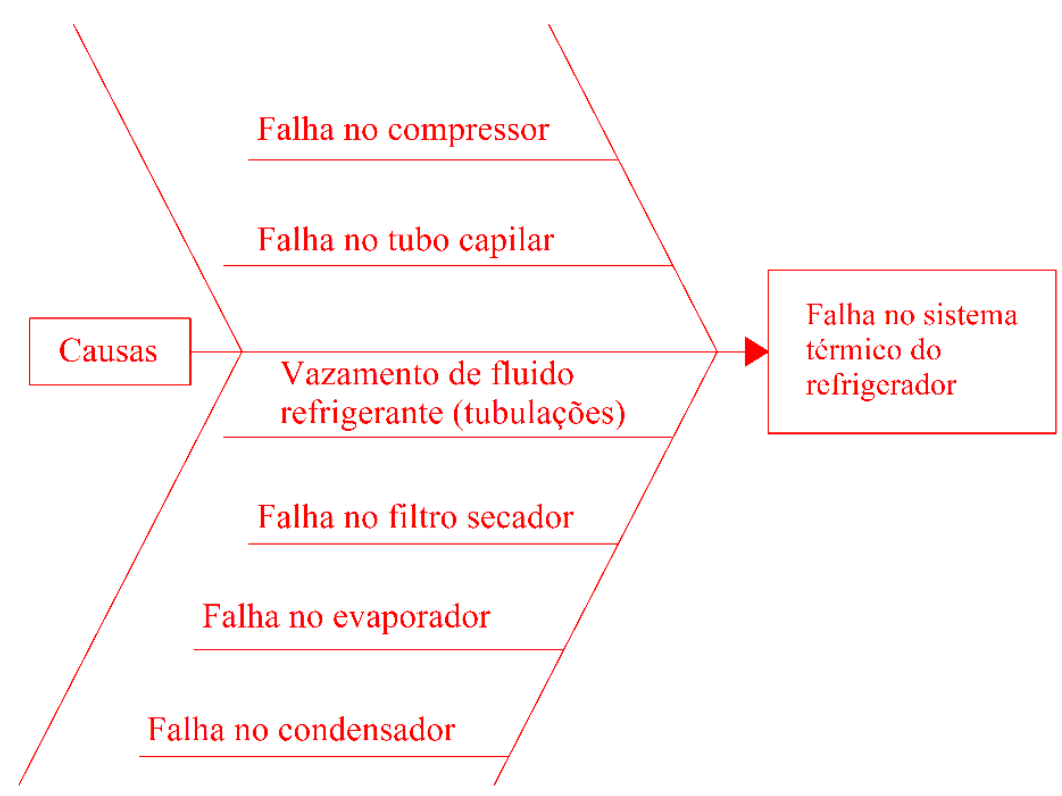

Figura 2 - Possíveis causas de falhas no compressor.

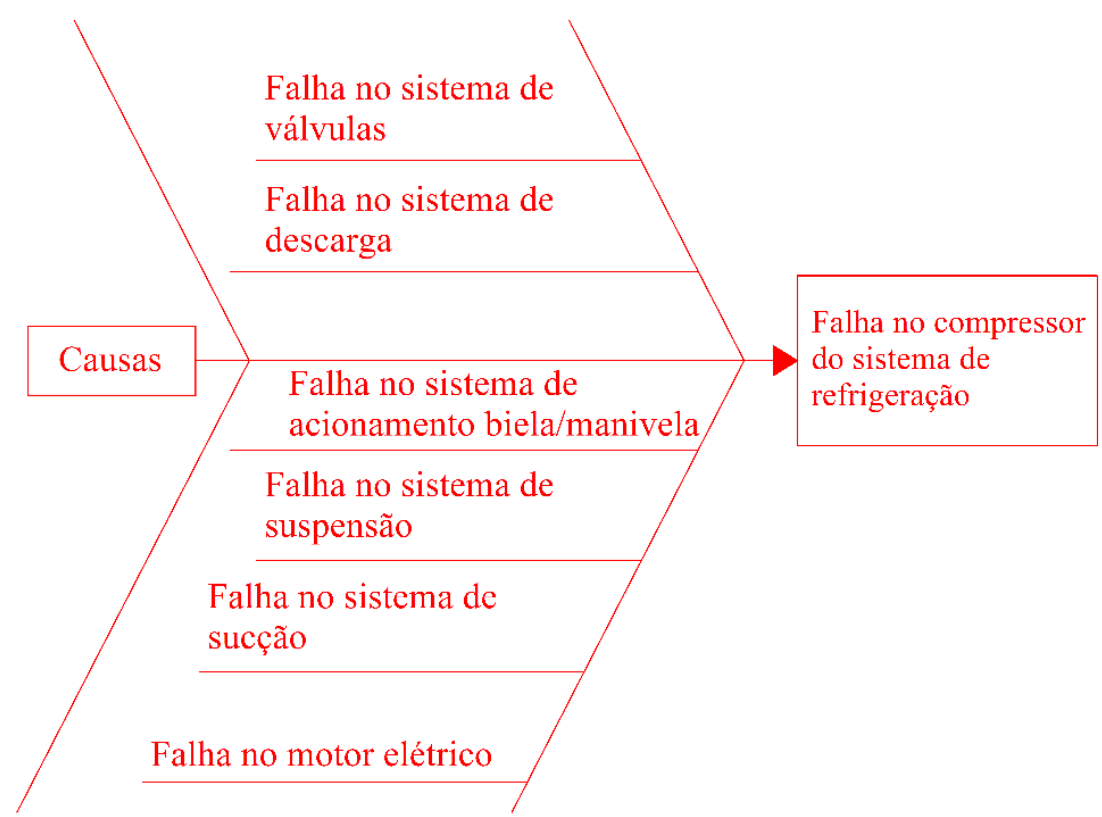

As falhas no compressor podem ser estudadas de acordo com o ciclo de vida do produto, falhas de início de ciclo estão relacionadas, geralmente, aos processos de projeto, fabricação e montagem. Falhas no tempo de vida útil podem estar associadas a enormes causas aleatórias, podendo ter origens no uso inadequado, transporte ou falhas relacionadas ao processo de fabricação e montagem que só ocorreram falha na fase de uso. Falhas relacionadas ao período de desgaste: geralmente estão associadas à fadiga dos componentes mecânicos, desgaste, entre outros. Como o estudo teve foco 
na manutenção do sistema de refrigeração, deu-se ênfase apenas em identificar a falha e substituir o compressor, já que o compressor é um sistema hermético concebido para funcionar todo o ciclo de vida sem manutenção.

Foram levantadas as principais causas de falhas no sistema de controle de um refrigerador doméstico. As causas foram selecionadas de acordo com a experiência do especialista na área. A Figura 3 mostra as causas de falhas no sistema de controle de um refrigerador doméstico.

Figura 3 - Possíveis causas de falha no sistema de controle.

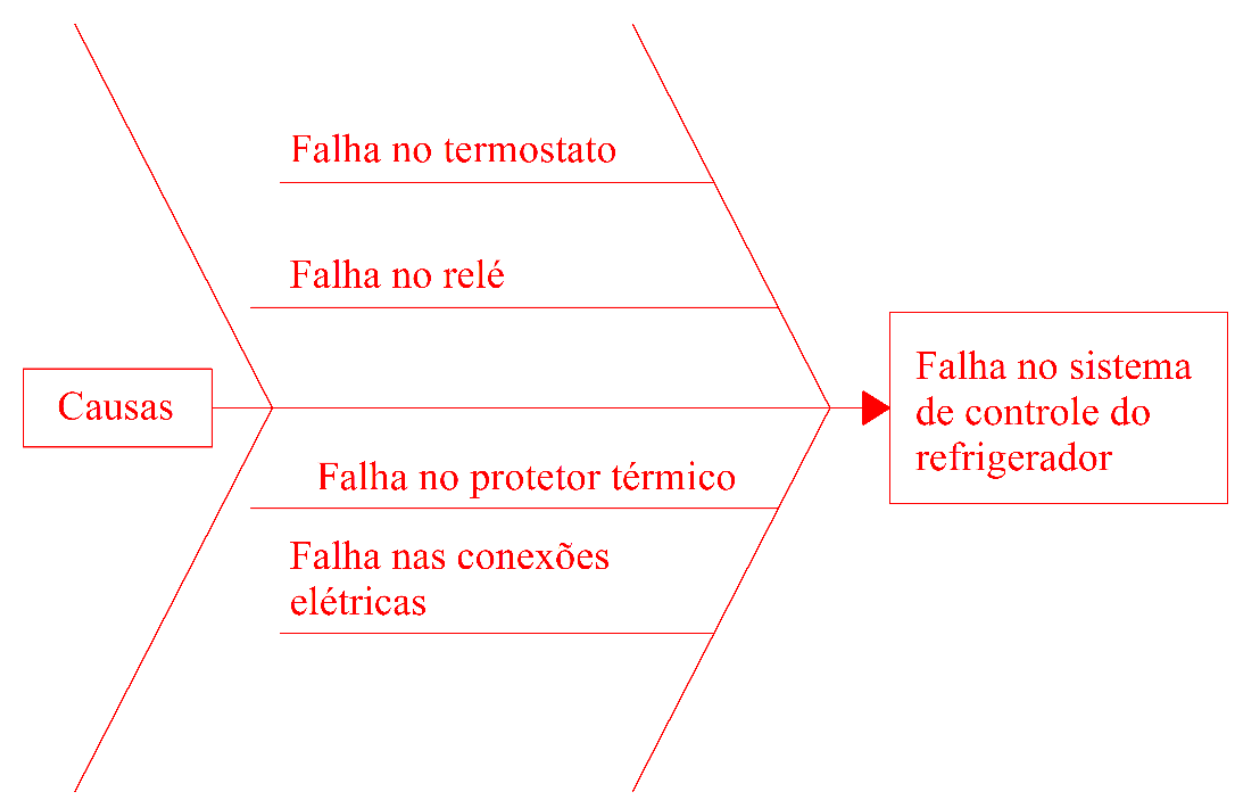

O termostato é o componente responsável por manter a temperatura do ambiente interno do refrigerador em uma faixa estabelecida pelo usuário, acionando ou desligando o compressor por meio do relê, faz isso utilizando um compartimento (bulbo) com um fluido de trabalho que expande e contrai com a temperatura, assim aciona uma membrana que comuta os contatos elétricos, uma falha no termostato pode comprometer todo sistema de refrigeração, pois o compressor não será acionado de maneira adequada. O relê é o dispositivo responsável por ligar e desligar o compressor de acordo com o sinal do termostato. O protetor térmico é o dispositivo responsável por proteger o compressor contra sobrecargas de corrente no sistema, falhas no protetor térmico podem deixar o sistema vulnerável a sobrecargas elétricas. As falhas no sistema de controle podem estar relacionadas às conexões elétricas, estas podem estar associadas a mau contatos, fios deteriorados e oxidação nos contatos elétricos.

Foram levantadas as possíveis causas de falhas devido ao sistema de isolamento, conforme Figura 4. As principais causas de falha foram consideradas como sendo devido à borracha da porta, falhas na porta interna do refrigerador e isolamento deteriorado. 
Figura 4 - Possíveis causas de falha no sistema de isolamento.

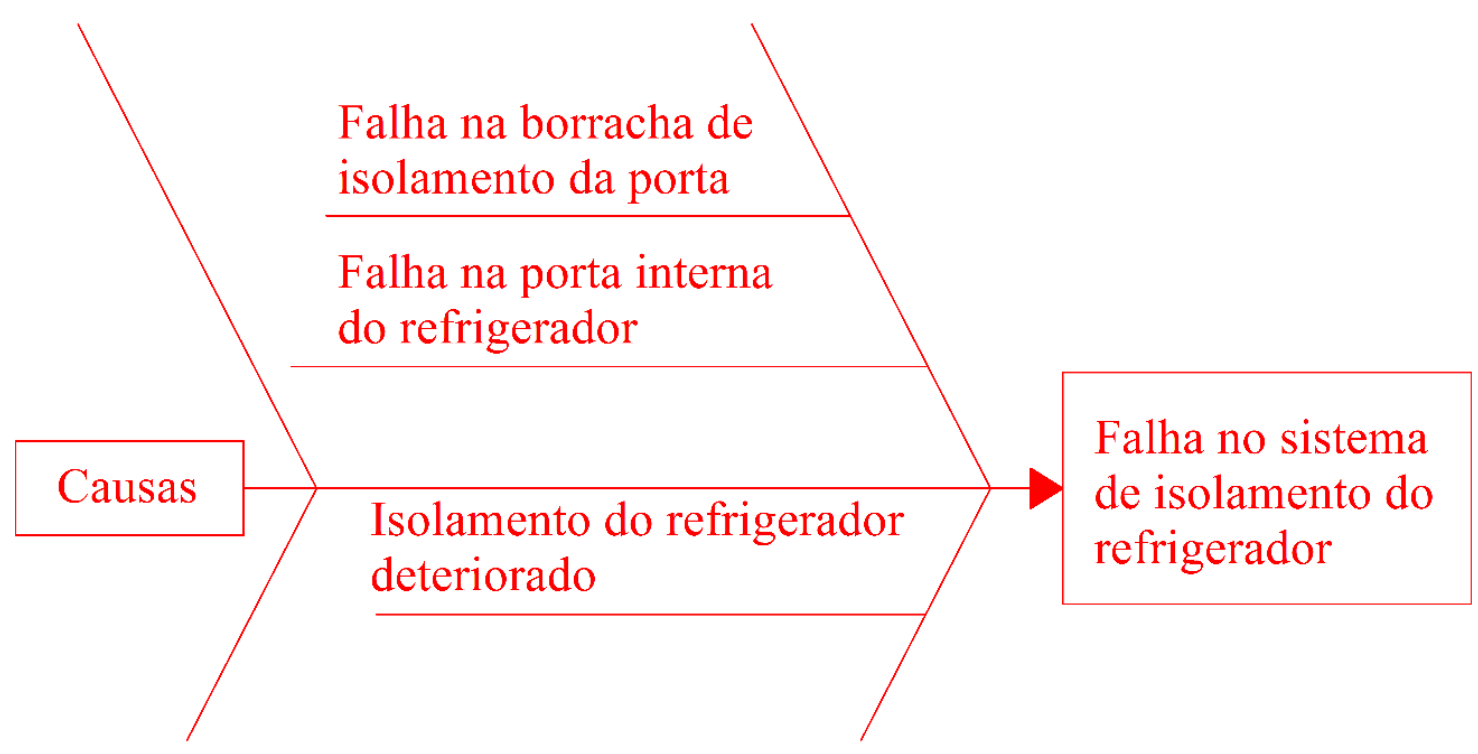

\subsection{FMEA do sistema de refrigeração}

Com objetivo de levantar os principais modos de falha a nível de sistema, utilizouse a técnica FMEA em um sistema de refrigeração genérico. A atenção principal foi a manutenção, nesse caso, procedeu-se uma análise a nível de sistema. O sistema técnico foi dividido em três partes básicas para análise, sistema térmico, sistema de controle e isolamento. O sistema térmico consiste basicamente nos componentes da máquina térmica, compressor, condensador, tubo capilar, tubulações, fluido refrigerante, filtro secador e evaporador. O sistema de controle é a parte do refrigerador responsável pelo controle da temperatura interna, cuja principal função é acionar o compressor ou desligá-lo, faz isso por meio dos componentes, termostato, relê conexões e protetor térmico. Por fim, o isolamento consiste basicamente em todo compartimento do refrigerador, paredes, revestimentos, porta interna e externa, os principais componentes utilizados na análise de FMEA foram a porta externa e interna, pois são os componentes que mais levam a falhas no sistema.

Observa-se que a análise realizada tem como objetivo identificar as causas básicas de falha no sistema de refrigeração doméstico, uma análise mais detalhada dos modos de falha dos itens do sistema não foi o escopo do estudo, pois, para um técnico em refrigeração, as causas raízes não são de grande relevância, pois uma vez identificadas as falhas básicas no sistema de refrigeração, o técnico irá substituir o componente defeituoso apenas. Por exemplo, o compressor possui vários subsistemas internos, a análise de FMEA pode ser realizada levando em conta vários aspectos, como, erros de montagem, erros de fabricação, falhas dos componentes, no final do ciclo de vida (fadiga e desgaste), entre outras, no entanto uma vez detectado qualquer modo de falha referente a qualquer fase do ciclo de vida, o técnico apenas substituirá o 
compressor, pois, como um sistema hermético não é concebido para ser manutenível, deve funcionar, durante todo o ciclo de vida, sem manutenções (DENCKER, 2002).

\subsection{Análise de Árvore de Falhas}

Para auxiliar a detecção de falhas utilizou-se, também, a técnica de FTA; no estudo, o sistema foi dividido em três componentes básicos novamente, máquina térmica, sistema de controle e sistema de isolamento. $O$ evento de topo na análise de FTA foi falha no sistema de refrigeração, tanto no contexto do FMEA quanto no FTA a falha é definida como qualquer desvio do sistema em cumprir a função programada.

A Figura 5 mostra a árvore de falhas geral do sistema de refrigeração. Observase que qualquer falha nos eventos intermédios levará à falha da geladeira, pois o sistema é conectado por portas lógicas do tipo "OU", o que representa um sistema série em que a confiabilidade é dada pelo produto de cada subsistema da máquina térmica.

Figura 5 - Árvore de falhas do sistema.

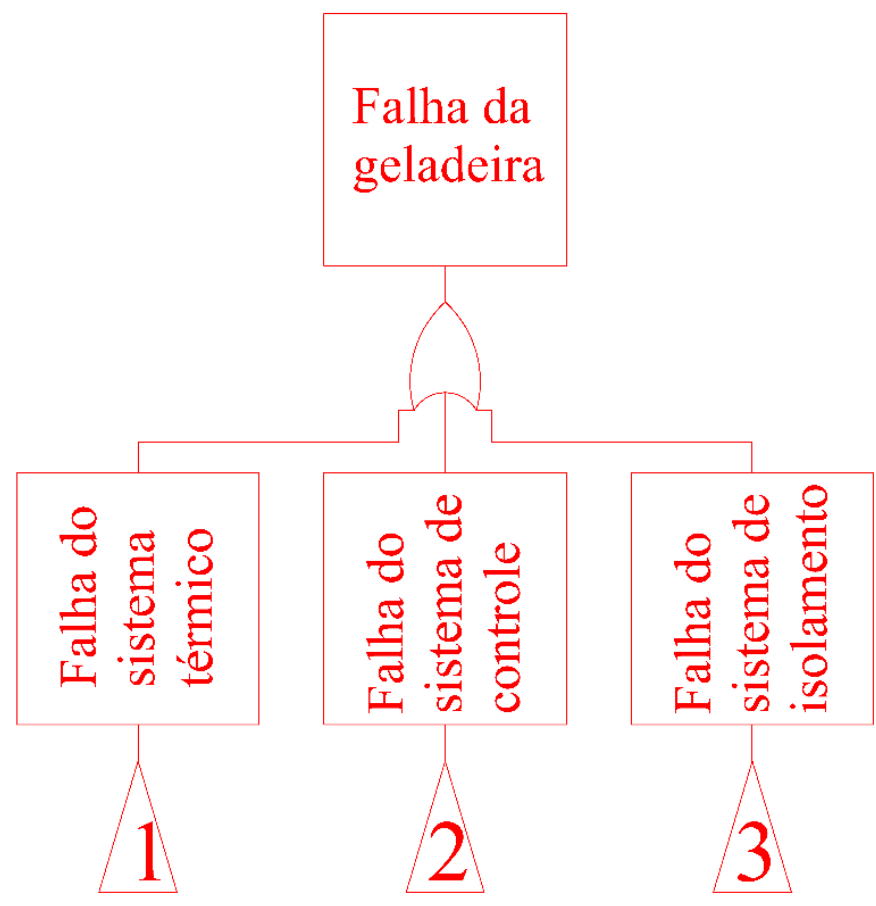

A Figura 6 mostra os eventos que levam à falha da máquina térmica, partindo-se do evento de topo falha no sistema térmico, existem seis eventos intermediários, os quais podem acarretar na falha do subsistema, como a falha no compressor; falha no condensador; falha no tubo capilar; falha no evaporador; falha nas tubulações e falhas no fluido refrigerante. Observa-se que o subsistema é conectado por portas lógicas do tipo "OU", o que significa que qualquer falha em um dos itens do subsistema acarretará 
em falha do sistema como um todo. Novamente este tipo de porta lógica é característica de sistemas séries, neste caso, a confiabilidade é dada pelo produto dos subsistemas, cada evento intermediário é conectado, ainda, por causas básicas ligados a portas lógicas "OU". Por exemplo, a falha no fluido refrigerante pode ser associada a causas raízes, presença de umidade no fluido refrigerante, volume de fluido refrigerante inadequado e, ainda, fluido refrigerante inadequado para o sistema. As causas básicas neste caso, estão associadas a erros de manutenção anteriores (não representadas na árvore), segundo Dias et al. (2011), as causas raízes, geralmente, estão relacionadas a aspectos organizacionais de uma empresa; ou seja, falha na manutenção, fabricação, projeto e montagem. Optou-se por não estender a análise a causas ainda mais básicas para sobrecarregar a árvore de falhas.

Na Figura 7, tem-se a análise do sistema de controle. Nota-se que é um sistema série, como evento de topo; neste caso, tem-se a falha do sistema de controle, os eventos intermediários são: falhas nas conexões elétricas; falhas no protetor térmico; falha no termostato e falha no relê. Cada um dos eventos intermediários é conectado por causas raízes ou outros eventos intermediários mais básicos. Observa-se que os itens do subsistema são conectados aos eventos ou causas raízes por portas lógicas "OU", característica de um sistema série. Por exemplo, partindo do evento intermediário falha no termostato, as causas raízes são, termostato inadequado para aplicação, fuga de gás do bulbo e oxidação dos contatos elétricos. 
Figura 6 - Árvore de falhas referente ao sistema térmico.

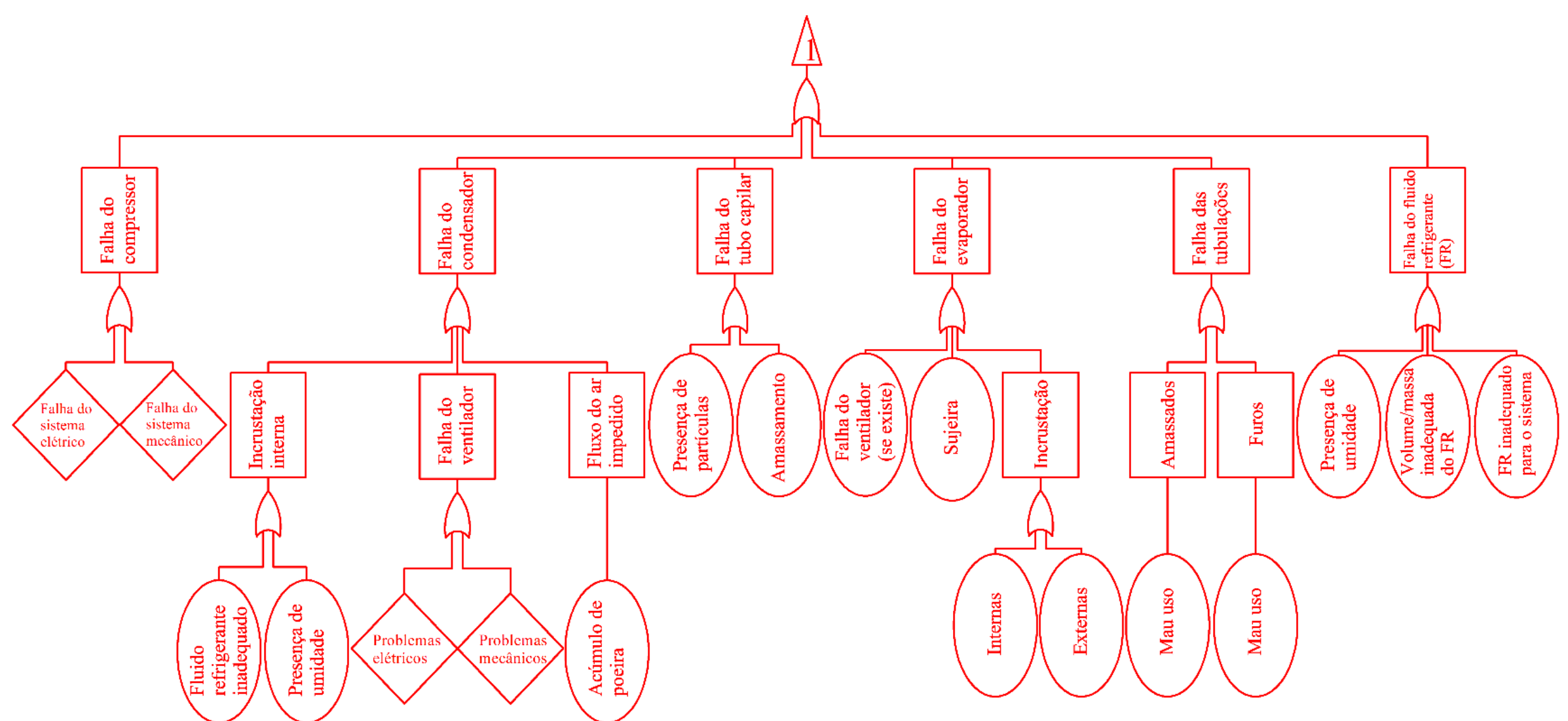


Figura 7 - FTA para o sistema de controle.

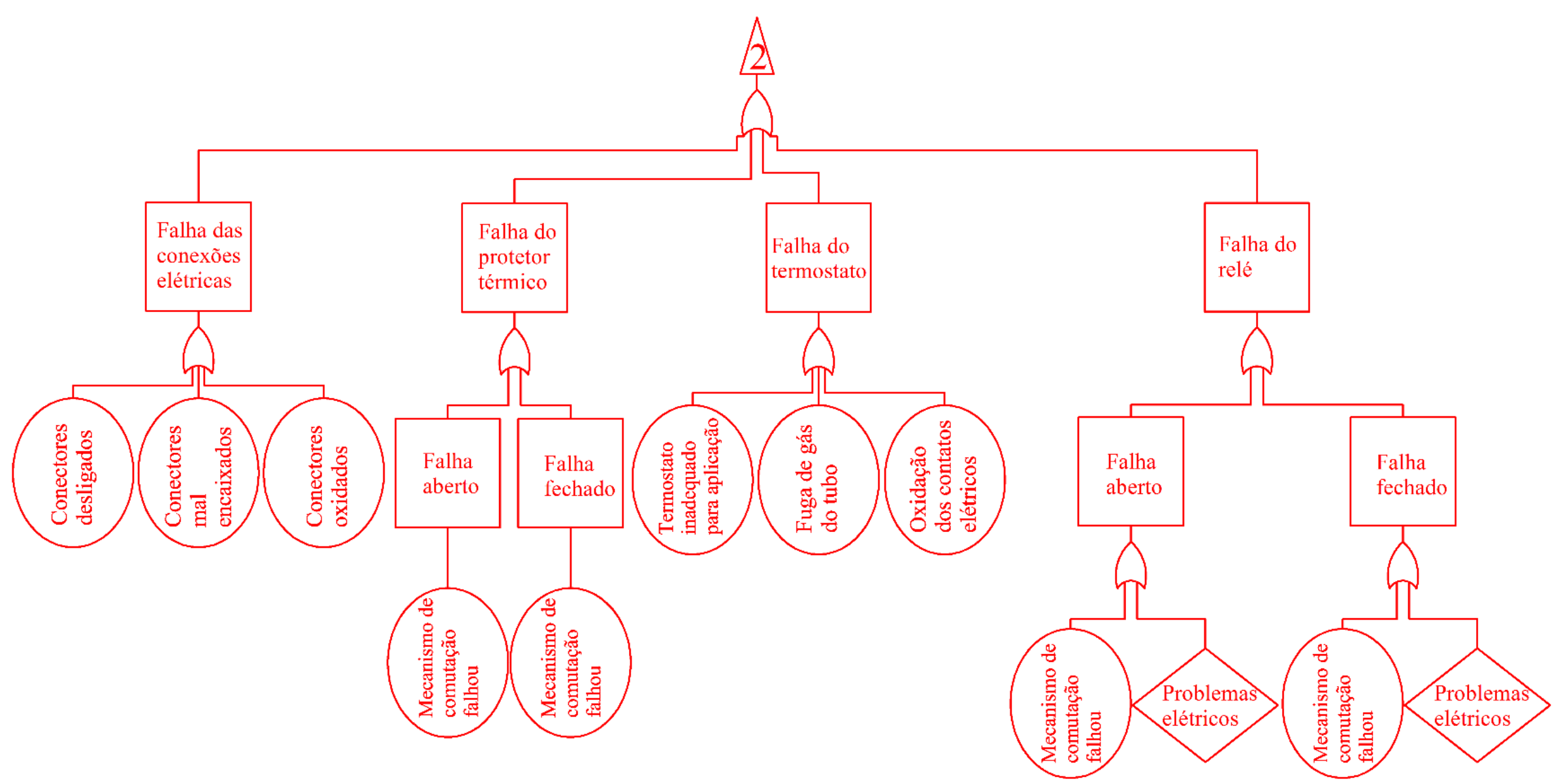


Por último, tem-se a árvore de falhas referente ao sistema de isolamento, conforme Figura 8. Neste caso, o evento de topo é a falha no sistema de isolamento, os eventos intermediários são problemas na porta interna e isolamento da porta externa do refrigerador, a falha no isolamento da porta externa, tem como causas raízes, borracha deteriorada e imã danificado.

Figura 8 - Árvore de falhas do sistema de isolamento.

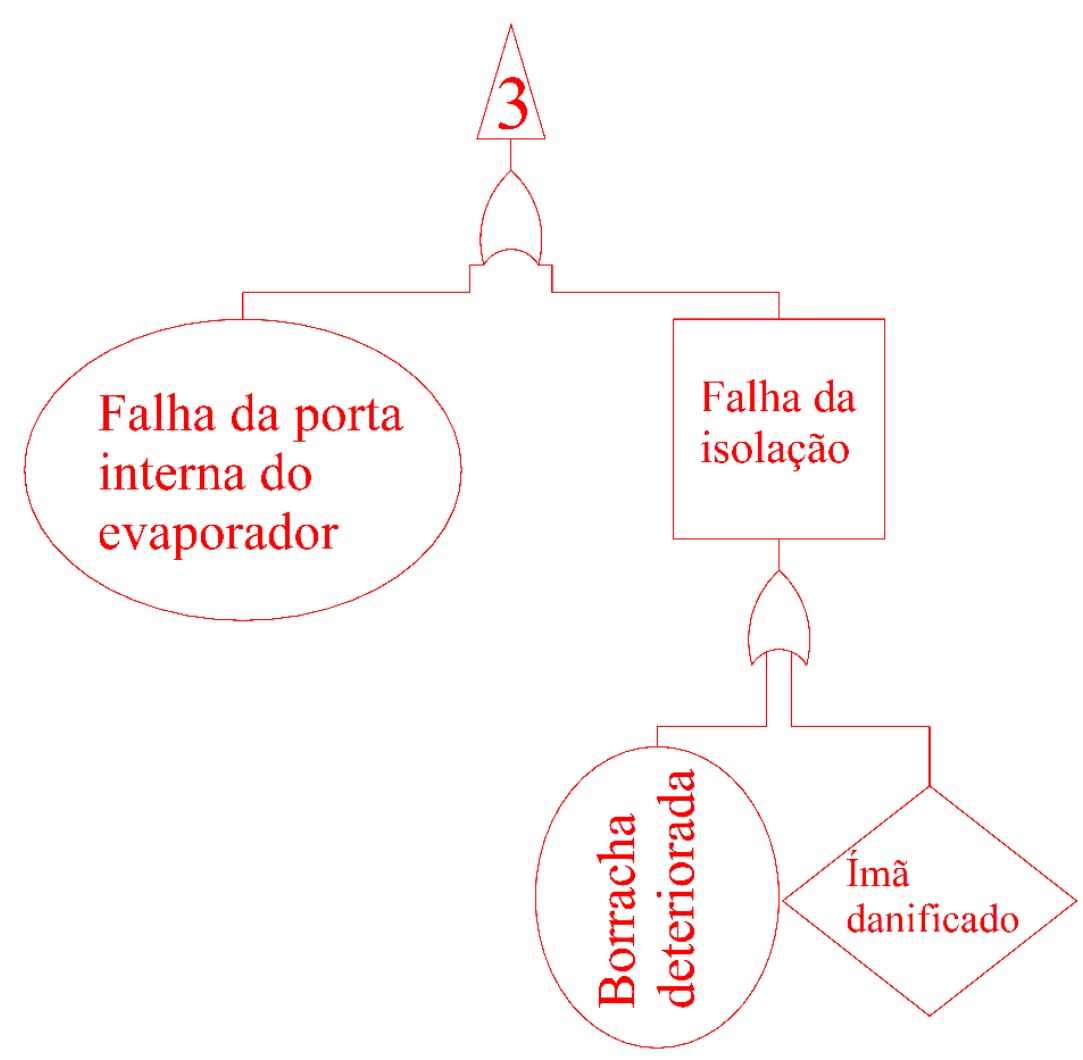

\section{RESULTADOS E DISCUSSÃO}

O protótipo final interage com o usuário por meio de perguntas, se é detectado falha em alguma parte do sistema, o SE mostra e explica os procedimentos de manutenção corretos. A pergunta inicial do sistema tem o caráter seletivo, tem o objetivo de fazer uma triagem entre as falhas que tornam o sistema inoperante (definidas como falhas) e as falhas que reduzem o rendimento do sistema (definidas como mau funcionamento). Diferentemente da definição de falha utilizada nas técnicas FMEA e FTA, o sistema faz distinção entre os tipos de falhas. A Figura 9 mostra o menu inicial do SE, existem duas opções para análise, falha e mau funcionamento.

Após a triagem inicial, o sistema realiza uma série de perguntas ao usuário e solicita que ele verifique partes do sistema a cada pergunta formulada; por exemplo, o SE pergunta ao usuário "o protetor térmico está funcionando?", e fornece a seguinte 
resposta "desligue o refrigerador, retire o protetor térmico do compressor, ligue o ohmímetro, entre os terminais. Assim, verifique o funcionamento do protetor térmico, teste as posições aberta e fechada, a posição aberta deverá dar continuidade de corrente e a fechada não deverá dar continuidade de corrente". Se a resposta for não, o sistema imprime o procedimento de manutenção; caso a resposta seja sim, o sistema passa para a próxima pergunta. Faz isso em uma sequência de testes, para identificar falhas, o sistema interage com o usuário, de forma semelhante ao médico com o paciente, faz perguntas e medições e afere um diagnóstico e tratamento.

Figura 9 - Exemplo de menu inicial do SE.

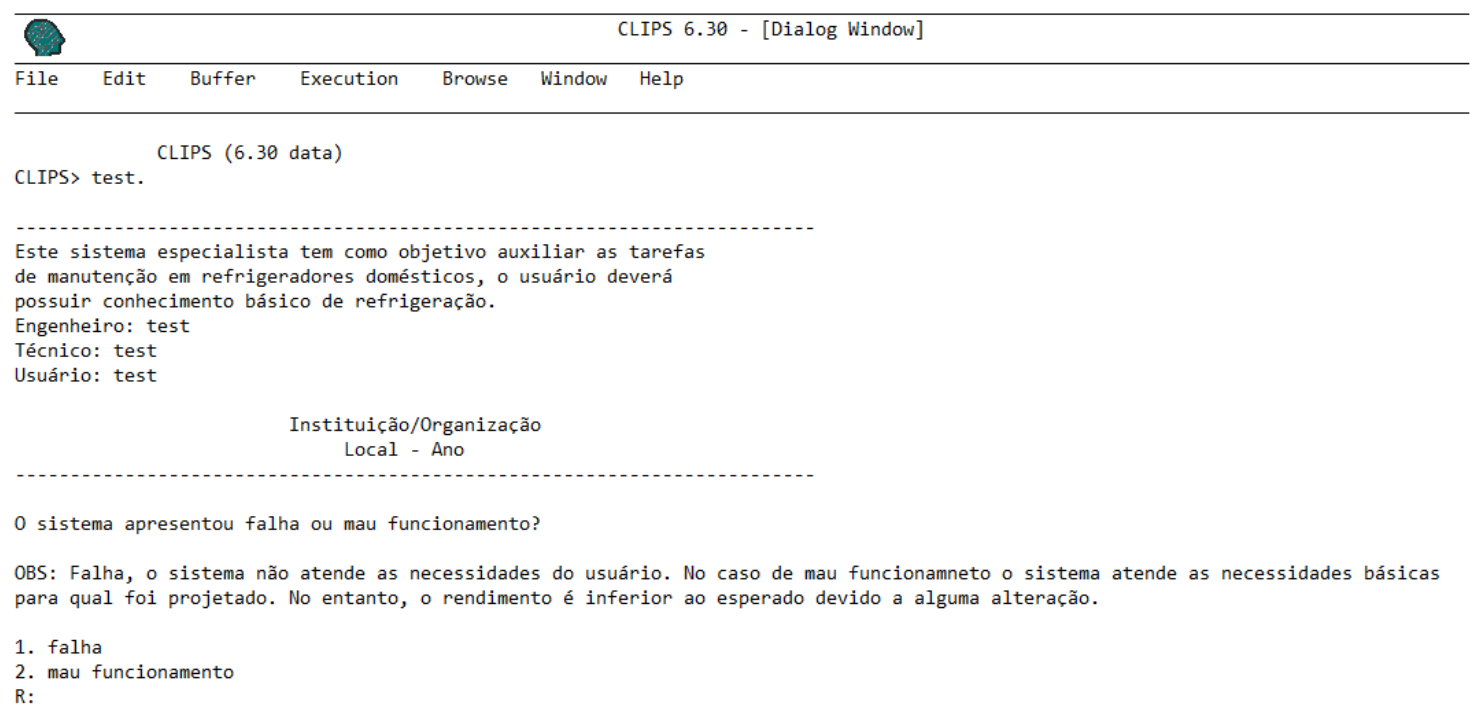

A Figura 10 mostra o fluxograma de como as perguntas são encadeadas para o usuário. Após a pergunta de triagem, a sequência de perguntas pode seguir o ramal direito ou esquerdo, o ramal direito representa o caso em que o sistema está em mau funcionamento, o ramal direito corresponde ao caso de falha, o protótipo inicial possui dez perguntas no ramal de falha e seis no ramal de mau funcionamento. $O$ corte, no fluxograma, significa que a estrutura se repete até a pergunta final, todas as perguntas com duas opções de saída, passar para próxima pergunta ou imprimir procedimento de manutenção, quando o sistema imprime o procedimento de manutenção existe mais duas opções, finalizar o programa ou reiniciar. Caso o SE passe por todas as perguntas sem encontrar falhas, a última oferece a opção de reiniciar ou finalizar o sistema. Quando o sistema não encontra falha, ele explica ao usuário que o SE é um protótipo e está em fase de teste e que, futuramente, pode se tornar mais preciso.

O sistema proposto por Feng et al. (2012), para diagnóstico de falhas em uma caixa de redução de uma turbina eólica, apresenta um sistema que interage com o usuário de forma diferente. O usuário escreve os "sintomas" da falha e o sistema lança as medidas corretivas. Dessa forma, o usuário não precisa passar por uma série de 
perguntas para encontrar um diagnóstico; no entanto, esse tipo de interação pode gerar erros de linguagem. Por exemplo, se o operador definir um sintoma como sendo eixo empenado e o usuário entrar com eixo torto, o SE não encontrará o diagnóstico adequado, o que pode gerar transtornos, ao usuário, maiores do que passar por uma série de perguntas restritas a respostas do tipo sim ou não. Por esse motivo, optou-se por uma interação por meio de perguntas.

Figura 10 - Fluxograma do SE

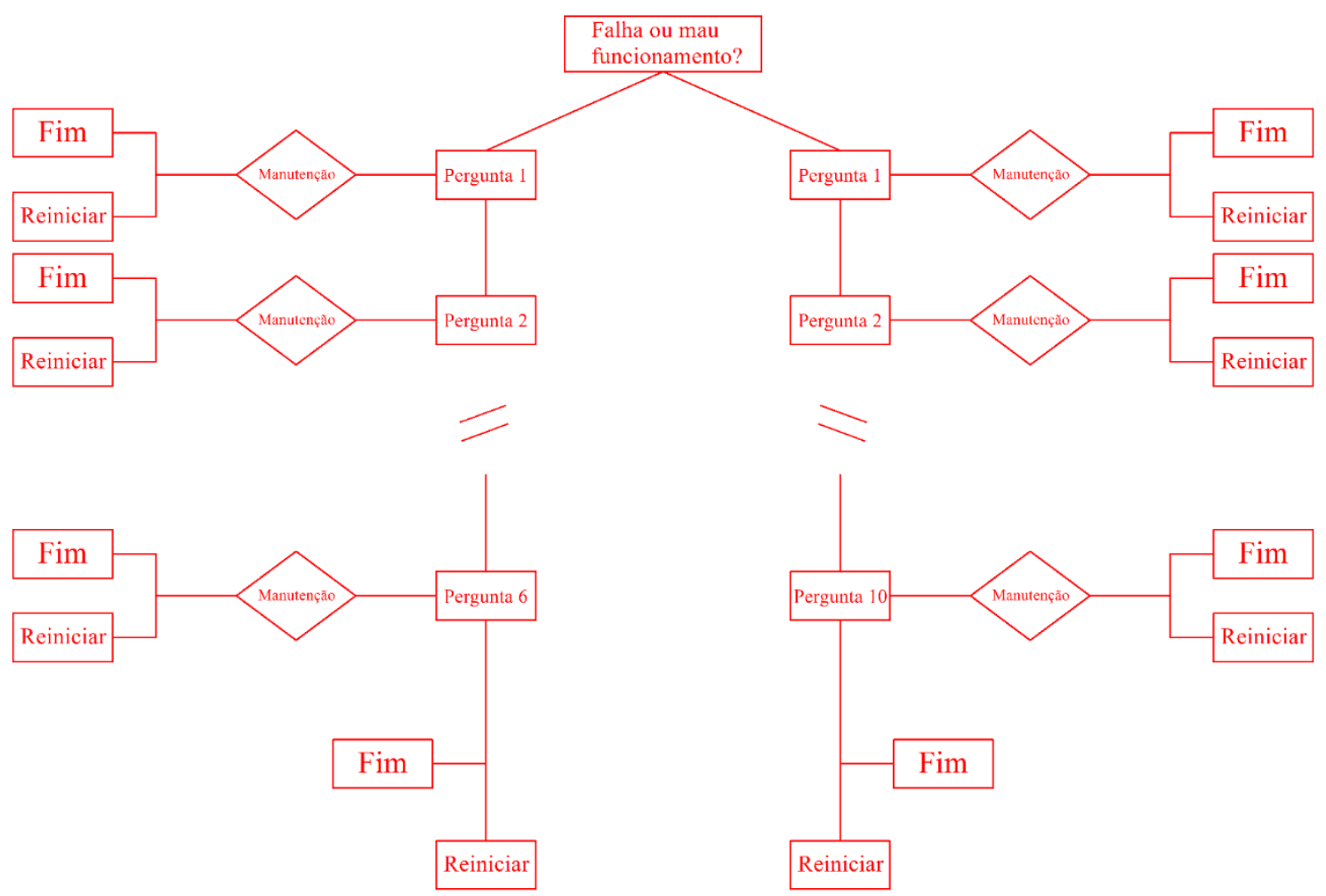

Mourão e Palmeira Junior (2011) propuseram um sistema com o objetivo de monitoramento em tempo real de um sistema de refrigeração industrial, o sistema proposto foi implementado em LABVIEW. O sistema opera com base em resultados de instrumentos e compara com o funcionamento considerado adequado para o sistema. Esse tipo de estrutura difere, totalmente, do sistema proposto neste trabalho, pois opera utilizando dados em tempo real, característica que foi considerada não viável em refrigeradores domésticos.

O SE proposto tem como foco servir de tutor inteligente para capacitação de técnicos em refrigeração, por esse motivo possui características mais informativas e gerais, não se restringindo a um tipo específico de refrigerador ou sistema de refrigeração. Toda análise em FMEA e FTA consideraram o refrigerador como um todo, sendo assim, análises mais detalhadas dos modos de falha de cada subsistema foram consideradas inadequadas por fugirem do escopo inicial. 


\section{CONCLUSÃO}

Sistemas especialistas são excelentes formas de gerir conhecimento e apoio pedagógico, além disso pode ser melhorado por incrementos de função, ou seja, tornarse cada vez mais preciso, detectando falhas no sistema. O sistema técnico é um sistema do tipo série do ponto de vista de confiabilidade, em que uma falha, em qualquer uma das partes, compromete todo o sistema. Por esse motivo, empresas que fabricam componentes do conjunto de refrigeração investem muito na confiabilidade de cada subsistema, como, por exemplo, o compressor.

\section{REFERÊNCIAS}

DENCKER, F. A. Detecção de falha na montagem de compressores herméticos por redes neurais artificiais. 2002. Dissertação (Engenharia Mecânica) Universidade Federal de Santa Catarina, Florianópolis, 2002.

DIAS, A. et al. Metodologia para Análise de Risco: Mitigação de Perda de SF6 em Disjuntores. 1. ed. Florianópolis: Editora Blumenau, 2011.

FENG, Y. et al. Monitoring wind turbine gearboxes. Wind Energy, v. 16, n. 5, 2013.

GIARRATANO, J. C.; RILEY, G. Expert Systems: principles and programming. Boston: PWS Publishing Co, 1994.

MOURÃO, M. S.; PALMEIRA JUNIOR, G. L. Desenvolvimento de um sistema de detecção e diagnóstico de falha aplicado em um sistema de refrigeração. 2011. Monografia (Engenharia Mecânica) - Universidade de Brasília, Brasília, 2011.

SILVA, J. C. Sistema especialistas aplicados à engenharia. Florianópolis: Programa de Pós-Graduação em Engenharia Mecânica da UFSC, 2015. [nota de aula].

Disponível em: <http://www.laship.ufsc.br/jonny/sist_esp/>. Acesso em: 10 abr. 2015. 